\title{
Characterization of Turbiditic Oil Reservoirs Based on Geophysical Models of their Formation
}

Semi-Annual Technical Progress Report

Reporting Period Start Date: July 1, 2004

Reporting Period End Date: September 30, 2004

Authors: Roger T. Bonnecaze and Srivatsan Lakshminarasimhan

August 2005

DOE Award \#: DE-FC26-01BC15352

Department of Chemical Engineering

The University of Texas at Austin 


\section{DISCLAIMER}

This report was prepared as an account of work sponsored by an agency of the United States Government. Neither the United States Government nor any agency thereof, nor any of their employees, makes any warranty, express or implied, or assumes any legal liability or responsibility for the accuracy, completeness, or usefulness of any information, apparatus, product, or process disclosed, or represents that its use would not infringe privately owned rights. Reference herein to any specific commercial product, process, or service by trade name, trademark, manufacturer, or otherwise does not necessarily constitute or imply its endorsement, recommendation, or favoring by the United States Government or any agency thereof. The views and opinions of authors expressed herein do not necessarily state or reflect those of the United States Government or any agency thereof. 


\section{Contents}

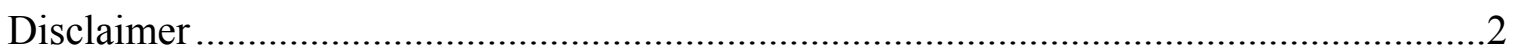

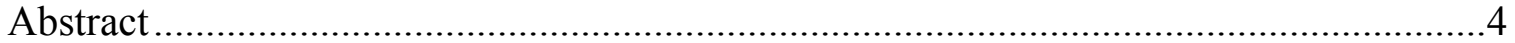

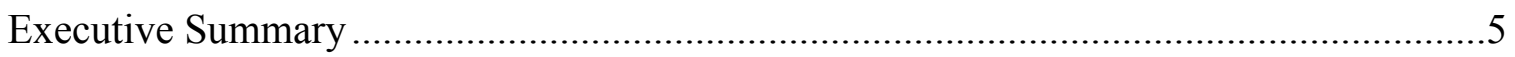

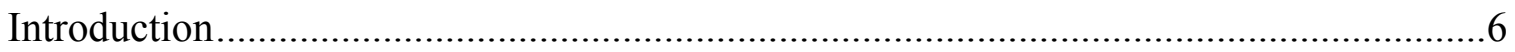

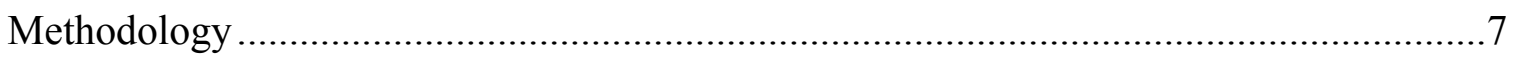

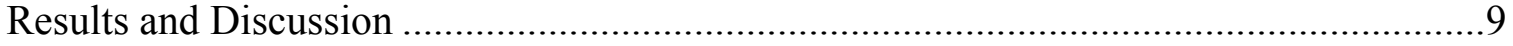

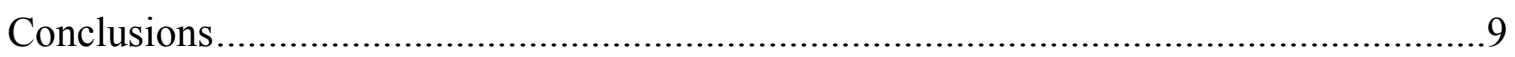

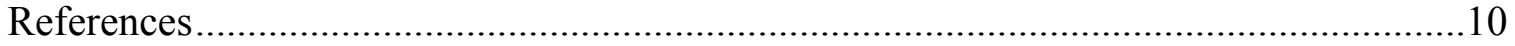




\begin{abstract}
The complete pseudo-three dimensional equations for the vertically averaged simplified suspension balance model (VASSBM) are presented for the simulation of the dynamics and deposition of concentrated turbidity currents. This simplified model is the result of the analysis of more exact equations for the flow and sedimentation process of turbidity currents. In previous reports we have presented the VASSBM model for twodimensional flows of constant volume or constant sediment flux. Here we generalize the equations to describe three-dimensional flows. We conclude with discussions on how the model may be implemented for oil reservoir characterization.
\end{abstract}




\section{EXECUTIVE SUMMARY}

In previous reports we have presented the exact Suspension Balance Model (SBM) and a variable grid FEM method for simulation of the SBM. The SBM equations are a good model of the turbidity flow but computationally expensive to simulate. Thus, we were motivated to produce the vertically averaged simplified suspension balance model (VASSBM) and validated it against the SBM equations for two-dimensional flows of constant volume or sediment flux. Here we present a generalized quasi-three dimensional version of the VASSBM equations which may be applied to viscous turbidity flow over arbitrary topography. It is quasi-3D because the equations involve vertically-averaged quantities, and the height of the current appears explicitly in the VASSBM.

The advantages of the VASSBM over the full SBM simulation are the following:

- VASSBM accurately captures all essential physics of the flow of turbidity currents,

- VASSBM contains a minimal number of independently determinable free constants,

- VASSBM is applicable to both hyperpycnal (constant sediment flux) as well as surge-type turbidity currents (constant volume),

- VASSBM is a 3D flows simulator

- VASSBM requires lower computation time than the SBM.

In summary the VASSBM is the superior model for simulation of the dynamics and deposition of turbidity currents that balances accuracy with computational speed. 


\section{INTRODUCTION}

The flow of oceanic turbidity currents often results in deposition of sediments. The deposited sediments, under propitious conditions, form oil-bearing reservoirs. Turbiditic hydrocarbon reservoirs constitute one of the most important sources of oil and gas in the near future. A reliable and cost-effective method to characterize turbiditic reservoirs is quintessential to optimum exploitation of such reservoirs. Geostatistical techniques are widely employed for this purpose with varying success. A method based on the physics of the formation of such turbidites can potentially augment, if not replace, geostatistical techniques.

The physics-based method or paradigm for reservoir characterization (PRC) can be divided into two parts. The first part, called the forward problem (FO), deals with calculation of deposition distribution, which results from a given turbidity current. The solution to the FP requires a process-based model for the flow of and deposition from turbidity currents. The second part, called the inverse problem (IP), consists of a procedure to predict the initial conditions of turbidity current(s) whose terminal deposition pattern match known data at specified locations.

The successful implementation of the new paradigm for reservoir characterization critically depends on the process-based model for turbidity currents. It is desirable to employ a process-based model which

- accurately captures all essential physics of the flow of turbidity currents,

- contains minimum number of independently determinable free constants,

- is applicable to both hyperpycnal as well as surge-type turbidity currents,

- is applicable to quasi-3D flows,

- requires nominally low computation time.

In the vertically averaged simplified suspension balance model (VASSBM), this work has presented a new process-based model with all the desirable properties listed above. Hence, the VASSBM is ideally suited for the new paradigm for reservoir characterization. This forms an important conclusion of this work.

The IP, which is the second part of the new paradigm for reservoir characterization, requires accurate prediction of the (1) nature and (2) initial conditions of turbidity currents that result in a given turbidite. Based on the characteristic features of a turbidite, it may be possible to predict the nature of the currents that resulted in the turbidite. Mulder \& Alexander (2001) presents some salient features of the turbidites that result from the flow of surge-type and hyperpycnal turbidity currents, which are given below:

- Surge-type turbidity currents tend to produce classical Bouma sequences. The particular nature of Bouma sequence found at any site can depend on flow size, sediment type, and proximity to source. 
- Hyperpycnal currents deposit coarsening-up units capped by fining up units because of waxing and waning conditions, respectively. the deposition can also include thick units of uniform character resulting from prolonged periods of nearly steady conditions.

The distinctive features of the turbidites formed by the two types of turbidity currents can help in the identification of the nature of currents which result in a given deposition pattern. However, the prediction of initial conditions of the currents which result in a given deposition requires detailed parametric analysis of the deposition pattern. Salient conclusions obtained from these analyses are presented later in this report.

The identification of a new grain support mechanism for turbidity currents in shear-induced particle migration (SIPM) is the final conclusion of this work. The phenomenon of SIPM accounts for migration of particles from regions of high shear stress to low shear stress. This enables upward movement of particles from the bottom regions of the current. thus, SIPM allows turbidity currents to retain particles for long duration and travel large distances even in the absence of turbulence.

The remainder of the chapter is organized as follows. The following section presents the salient features of the VASSBM.

\section{METHODOLOGY}

\section{VASSBM: A new process-based model}

The salient features of the VASSBM are given below:

- The VASSBM is obtained from the suspension balance model (SBM). The SBM is a rigorous physics-based model for turbidity currents. By retaining all the important features of the SBM, the VASSBM captures all essential physics of the flow of turbidity currents. In particular, the VASSBM captures the effects of both the dimensionless numbers $\left(\phi_{i n}\right.$ and $\left.N_{B}\right)$ on the flow of and deposition from turbidity currents.

- The VASSBM contains only one free constant in $k_{N}$, which can be determined independently through experiments (Morris \& Boulay, 1999).

- The VASSBM was validated for both hyperpycnal as well as surge-type turbidity currents. Hence, it is applicable to both types of currents.

- The VASSBM can be extended to quasi-3D flows as well. A derivation of VASSBM equations for quasi-3D flows is presented in this section.

- The numerical solution to VASSBM equations up to $t=100$ typically requires about 15 minutes of computation time. On the other hand, the computation time required to solve the complex SBM equations up to $t=20$ is typically about 15 hours! The need for nominally low computation time makes VASSBM wellsuited for long-time calculations. 
Clearly, the VASSBM meets all the properties desired in a process based model for turbidity currents. Thus, the VASSBM is ideally suited as the process based model to study the flow of and deposition from turbidity currents.

The VASSBM was obtained by integrating the equations of the simplified suspension balance model (SSBM) in the vertical direction. The SSBM equations presented in this report are applicable only to 2D-flows. However, the SSBM as well as VASSBM, can be extended to quasi-3D flows. The SSBM equations for threedimensional flows are presented below:

$$
\begin{gathered}
\nabla \square\langle\boldsymbol{u}\rangle=0, \\
-\left(\rho_{p}-\rho_{f}\right) g \phi \boldsymbol{e}_{y}-\nabla P+\eta \frac{\partial}{\partial y}\left[\left(\eta_{\mathrm{s}} \frac{\partial\langle u\rangle}{\partial y}\right) \boldsymbol{e}_{x}+\left(\eta_{\mathrm{s}} \frac{\partial\langle w\rangle}{\partial y}\right) \boldsymbol{e}_{z}\right]=0, \\
\frac{\partial \phi}{\partial t}+\nabla \square(\phi\langle u\rangle)=-\frac{2 \mathrm{a}^{2}}{9 \eta} \nabla \square[f(\phi)(\nabla P-\nabla \Pi)] \\
P=p_{f}+\Pi, \quad \Pi=\eta \eta_{n}(\phi) \dot{\gamma}
\end{gathered}
$$

Here, $\langle\boldsymbol{u}\rangle \equiv(\langle u\rangle,\langle v\rangle,\langle w\rangle)$ represent suspension velocity in stream-wise (x), vertical (y) and span-wise $(z)$ directions, respectively. The vertically integrated form of the above 3D-SSBM equations (3D-VASSBM) are given below:

$$
\begin{gathered}
F(h, \phi)=\frac{\left(\rho_{p}-\rho_{f}\right)}{\eta} \frac{1}{\eta_{\mathrm{s}}(\phi)}\left[\frac{1}{6} \phi h^{3} \frac{\partial h}{\partial x}-\frac{1}{8} \frac{\partial}{\partial x}\left(\phi h^{4}\right)\right], \\
G(h, \phi)=\frac{\left(\rho_{p}-\rho_{f}\right)}{\eta} \frac{1}{\eta_{\mathrm{s}}(\phi)}\left[\frac{1}{6} \phi h^{3} \frac{\partial h}{\partial z}-\frac{1}{8} \frac{\partial}{\partial z}\left(\phi h^{4}\right)\right], \\
\frac{\partial h}{\partial t}+\frac{\partial F}{\partial x}+\frac{\partial G}{\partial z}=0, \\
\frac{\partial}{\partial t}(\phi h)+\frac{\partial}{\partial x}(\phi F)+\frac{\partial}{\partial z}(\phi G)=-v_{\mathrm{s}} \phi f(\phi) \\
-\frac{k_{N}}{\phi_{m}^{2}} v_{\mathrm{s}} \phi^{2} f(\phi)\left\{\left[\frac{\partial(\phi h)}{\partial_{x}}\right]^{2}+\left[\frac{\partial(\phi h)}{\partial_{z}}\right]^{2}\right\}^{1 / 2} .
\end{gathered}
$$




\section{RESULTS AND DISCUSSION}

\section{Parametric analysis using VASSBM}

The VASSBM enabled the calculation of the length and average thickness of deposition pattern resulting from hyperpycnal as well as surge-type turbidity currents at long times. The dimensions of the deposition were obtained for a range of initial particle concentration $\left(0.01 \leq \phi_{\text {in }} \leq 0.4\right)$ and buoyancy number $\left(0.01 \leq N_{B} \leq 0.2\right)$ values. The following conclusions are drawn from these studies.

- The length of deposition $\left(l_{d e p}\right)$ is marginally influenced by the buoyancy number $N_{B}$. This highlights a competition between two contrasting issues. Firstly, increase in $N_{B}$ signifies bigger or heavier particles which correspond to increase in the buoyancy of the current. Secondly, increase in $N_{B}$ also enhances settling which decreases volume of suspended particles and hence the buoyancy of the current. This behavior is seen in the deposition pattern obtained from both hyperpycnal as well as surge-type currents.

- The length $l_{\text {dep }}$ is significantly influenced by $\phi_{i n}$ Yet, the variation of $l_{d e p}$ with $\phi_{i n}$ highlights a competition. Firstly, increase in $\phi_{i n}$ increases viscosity of suspension $\left(\eta_{\mathrm{s}}\right)$ which decreases horizontal flux of the current. Secondly, increase in $\phi_{\text {in }}$ increases the buoyancy of the current which increases horizontal flux. In the case of hyperpycnal currents, the former effect is pronounced at low $N_{B}$ than the latter. Hence, the $l_{\text {dep }}$ from hyperpycnal currents decreases with $\phi_{i n}$ at low $N_{B}$ and vice vers $a$ at high $N_{B}$. On the other hand, the $l_{\text {dep }}$ from surge-type currents show nonmonotonic variation with $\phi_{i n}$ at all $N_{B}$ values in the considered range.

- The average thickness of deposition $t_{\text {dep }}$ monotonically increases with $N_{B}$. This is explained by the increase in settling velocity of particles with $N_{B}$. The average thickness obtained from hyperpycnal currents scales $t_{\text {dep }} \square N_{B}^{0.9}$ which approximates the theoretical scaling of $t_{d e p} \square N_{B}$. The average thickness obtained from surge-type currents, however, do not show any clear scaling with $N_{B}$.

- The average thickness is influenced by $\phi_{i n}$, which highlights a competition between two issues. Increase in $\phi_{i n}$ increases the total volume of suspended particles which increases deposition. On the other hand, increase in $\phi_{\text {in }}$ decreases settling velocity of particles which decreases deposition.

\section{CONCLUSIONS}

The quasi-3D vertically averaged SSBM (3D-VASSBM) equations (5) through (8) retain all essential features of the physics of the flow of viscous turbidity currents. In 
particular, it captures the effect of shear induced particle migration which enables viscous turbidity currents to travel long distances. The good agreement between the results obtained from vertically averaged SSBM (VASSBM) with the corresponding results obtained from the rigorous SBM demonstrates the reliability of VASSBM. Even though VASSBM is physics based, it is simple enough to enable calculation of deposition thickness within a few minutes of computation time. The reliability and ease of use of VASSBM makes it ideally suited as the process-based model for turbidity currents.

The derivation of the rigorous SBM for the study of viscous turbidity currents, which enabled the derivation of VASSBM, represents a high point of this work. The development of SBM required a thorough understanding of the fundamental physics of the flow of viscous turbidity currents. The model takes into account the dual role played by buoyancy, the hydrodynamic drag experienced by particle phase due to relative motion between particle and suspension phases, and shear induced migration of particles. The shear-induced migration enables upward motion of particles. This feature of the flow enables viscous turbidity currents (particularly hyperpycnal flows) to travel large distances. The SBM can be extended to include inertial effects. The extended SBM (ESBM) can be used to study turbulent turbidity currents.

The SBM consists of a set of non-linear and coupled partial differential equations with variable coefficients. The variable viscosity coefficients diverge to infinity in regions of deposition. Consequently, the numerical algorithm to solve the SBM equations is complex and involved. The development of the FEM-based algorithm represents a significant achievement of this work. The FEM-based algorithm enabled computationally efficient and accurate simulations of the dynamics of and deposition from turbidity currents.

In addition to the study of highly concentrated viscous turbidity currents, this report also presents a study of low concentration turbulent turbidity currents through scaling analysis. This is the first time the method of scaling analysis has been applied to a rigorous turbidity current model such as ESWM. The estimates for dimensions of deposition which were obtained through application of scaling analysis, as well as the method itself, can benefit sedimentologists and field geologists.

\section{REFERENCES}

Morris, J. F., and Boulay, F. (1999) "Curvilinear flows of noncolloidal suspensions: The role of normal stresses,” J. Rheol., 43, 1213-1237.

Mulder, T., and Alexander, J. (2001) "The physical character of subaqueous sedimentary density flows and their deposits," Sedimentology, 48, 2, 269-299. 